\title{
X-RAYS FROM PHOTOIONIZED ACCRETION DISCS
}

\author{
G. MATT and A.C. FABIAN \\ Institute of Astronomy, Madingley Road, Cambridge, CBS OHA, U.K. \\ and \\ R.R. ROSS \\ Physics Department, College of the Holy Cross, Worcester, MA 01610, USA
}

The presence of iron lines and high energy excesses in the X-ray spectra of Seyfert galaxies has been firmly established by Ginga (e.g. Nandra \& Pounds 1993 and references therein). These features are generally interpreted as signatures of the reprocessing of the primary X-rays by matter in the neighbourhood of the central black hole, probably distributed in an accretion disc (Lightman \& White 1988, George \& Fabian 1991, Matt, Perola \& Piro 1991).

The illumination of the disc by the X-rays can significantly alter the thermal and ionization structure of the surface layers, modifying the lines and continuum emission properties. We have studied in detail these effects; the main results can be summarized as follows:

i) While for low-ionization matter the only important line in the X-ray band is the iron fluorescence one, many other lines are expected from highly ionized matter (Ross \& Fabian 1993).

ii) The properties of the iron line itself are modified if the matter is ionized. The fluorescence yield increases with the ionization state, and the importance of reabsorption diminishes with the ionization of the matter. On the other hand, the FeXVII-FeXXIII line photons are destroyed by resonant trapping. Therefore, the line intensity can be either much smaller or much greater than for the neutral case (Ross \& Fabian 1993, Matt, Fabian \& Ross 1993a,b).

iii) If the matter is highly ionized the disc could be significantly reflective even in the soft X-rays. The resulting emerging continuum spectra are quite complex, and have been calculated in great detail for a single slab by Ross \& Fabian (1993). Matt, Fabian \& Ross (1993c) extended the calculation to the whole disc, and estimated the angular dependence on the flux. They also calculated the polarization; both the degree and the angle resulted to be strongly energy-dependent.

\section{References}

George I.M., Fabian A.C., 1991, MNRAS, 249, 352

Lightman A.P., White T.R., 1988, ApJ, 335, 57

Matt G., Fabian A.C., Ross R.R., 1993a, MNRAS, 262, 179

Matt G., Fabian A.C., Ross R.R., 1993b, MNRAS, submitted

Matt G., Fabian A.C., Ross R.R., 1993c, MNRAS, in press

Matt G., Perola G.C., Piro L., 1991, A\&A, 247, 25

Nandra K., Pounds K.A., 1993, MNRAS, submitted

Ross R.R., Fabian A.C., 1993, MNRAS, 261, 74

380

T. J.-L. Courvoisier and A. Blecha: Multi-Wavelength Continuum Emission of AGN, 380.

(C) 1994 IAU. Printed in the Netherlands. 